\title{
45. SPECTRAL CLASSIFICATIONS AND MULTI-BAND COLOUR INDICES \\ (COMMISSIONS DES CLASSIF ICA TIONS SPECTRALES ET INDICES DE COULEUR A PLUSIEURS BANDES)
}

PRESIDENT: C. Jaschek

VICE-PRESIDENT: E.K. Kharadze

ORGANIZING COMMITTEE: A.P. Cowley, B. Hauck, W. Iwanowska, P.C. Keenan, E. E. Mendoza, V. Sinnerstad, V. Straizys and B. E. Westerlund.

\section{INTRODUCTION}

The period covered in this report $(1973-75)$ is one of exceptional activity in classification, in all its different aspects. During this period the extreme ultraviolet region has become accessible to classification techniques through observations from space. Large projects, like the re-classification of the HD stars by means of two dimensional schemes, have been started. New techniques hold a promise to produce an advance to fainter magnitudes. Many new catalogues have been published, permitting an easy access to data scattered otherwise throughout the literature. Automated techniques begin to appear as a substitute for time-consuming human work. Theory has advanced far enough to interpret the many subtleties found in photometric observations. Much of this progress has been reported in the meetings held during this period. We will mention just the most important ones.

The Albany Colloquium, on 'Multicolor photometry and the theoretical HR diagram' held in October 1974, published as Dudley Observatory Report No. 9 (ed. by Philip and Hayes) (1975), provides a large number of important contributions.

IAU Symp. 72, on 'Abundance Problems in Classification' was held in July 1975 at Lausanne. It was dedicated to $\mathrm{Dr}$ W. W. Morgan, in recognition of his outstanding contributions to astronomy. The proceedings are edited by Keenan and Hauck. An important feature of the symposium were the several general review papers.

IAU Colloq. 32, on the 'The Physics of Ap Stars', held in Vienna, September 1975, dealt also with some matters concerning our Commission, as did the Twentieth Liège International Astrophysical Colloquium, held in June 1975, on 'Astrophysics and Spectroscopy'.

Due to the increasing number of scientists and of papers, I have felt that it is best to divide the Report in four main sections, each written by a different person, with myself responsible for the final editing. At the end, Dr B. Hauck, President of the working group on Spectroscopic and Photometric Data, presents the Report of the group. It is to be regretted that not all Commission members did report on their work, a fact which is responsible for some of the omissions which might occur.

\section{WORK INVOLVING SLIT SPECTROGRAMS \\ (W. P. Bidelman)}

There has been much activity in the classification of stellar spectra utilizing slit spectrograms, mainly, but not exclusively, directed toward application of the MK system. It seems impossible to adequately summarize this work; thus I have simply listed in the following most of the relevant papers that have appeared in the period mid-1972 to mid-1975. Some general papers and others of a more fundamental nature are indicated by asterisks. There are a number of important exclusions: papers presented at IAU Symposia are not included, nor are papers dealing with the classification of only a few stars or ones that, though containing data of importance to spectral classification, are not primarily directed toward that end. It was also not 
considered feasible to list papers dealing with the classification of the spectra of galaxies and planetary nebulae. A stronomy and Astrophysics Abstracts' numbers are added for reference. A few items communicated to the writer are given at the end of the list.

\section{REFERENCES}

Abt, H. A. (13.153.030); Abt, H. A. and Moyd, K. I. (9.114.170); Adelman, S. J. (10.152.010); Balona, L. A. (13.114.001); Balona, L. and Crampton, D. (11.115.002); Barbier, M. (13.114.017); Barbon, R. and Hassan, S. M. (9.153.015); Barry, D. C. and Cromwell, R. H. (11.153.001); Bernacca, B. L. and Ciatti, F. (8.114.016); Bond, H. E. and Tifft, W. G. (12.122.129); Bonsack, W. K. (12.114.062); Boulon, J., Doazan, V., and Letourneur, N. (12.114.008); Boulon, J., Doazan, V., and Letourneur, N. (13.114.354); Brandi, E. and Clariá, J. J. (10.114.033); Brunet, J.-P., Prévot, L., Maurice, E., and Muratorio, G. (9.114.045); Carozzi, N. (12.112.003); Chalonge, D. and Divan, L. (9.115.003); *Conti, P. S. and Leep, E. M. (12.114.083); Cowley, A. (8.114.109); Cowley, A. (9.114.174); Cowley, A. and Fraquelli, D. (11.114.063); Coyne, G. V. (8.115.013); Crampton, D. (8.112.002); Crampton, D. and Evans, T. L. (9.114.098); Crampton, D. and Fisher, W. A. (13.1 14.086); Crampton, D., Leir, A., and Younger, F. (11.114.170); Dachs, J. (8.114.113); Delplace, A. M. and Hubert, H. (13.114.010); Dickens, R. J., Feast, M. W., and Evans, T. L. (8.122.044); Drilling, J.S. and Pesch, P. (9.113.019); DuPuy, D. L. (10.122.080); Dworetsky, M. M. (13.153.005); Erro, B. I. Bull. Inst. Tonantzintla, 1, 209, 1975; Fawley, W. M. and Cohen, M. (12.153.026); Feast, M. W. (12.122.043); Fehrenbach, Ch. (12.112.004); Garrison, R. F. (8.153.026); Glaspey, J. W. (8.152.001); Graham, J. A. and Slettebak, A. (9.1 14.127); Greenstein, J. L. (11.126.019); Greenstein, J. L. (12.126.025); Greenstein, J. L. (13.126.006); *Greenstein, J. L. and Minkowski, R. (9.125.030); *Greenstein, J. L. and Sargent, A. I. (12.1 14.061); Gulliver, A. F. and MacRae, D. A. (13.114.051); Harlan, E. A. (11.114.152); Henize, K. G. and Mendoza, E. E. (9.152.003): Herbst, W. (13.1 32.018); Hill, G., Fisher, W., and Allison, A. (11.153.012); Hill, G., Hilditch, R.W., Younger, F., and Fisher, W. A., Mem. Roy. Astron. Soc. 79, 131, 1975; Hill, P. W., Kilkenny, D., and van Breda, I. G. (12.113.012); Hintzen, P. and Strittmatter, P. A. (12.126.023); Hubert, H. (9.114.013); Humphreys, R. M. (9.114.012); Humphreys, R. M. (11.114.162); Humphreys, R. M. (13.114.013); Humphreys, R. M. and Ney, E. P. (12.114.150); Irvine, N. J. (13.114.022); *Jaschek, M. and Jaschek, C. (12.114.119); *Jaschek, M. and Jaschek, C. (12.114.075); Jones, D. H. P. $(8,155.055)$; Joy, A. H. and Abt, H. A. (12.114.035); *Keenan, P.C., Garrison, R. F., and Deutsch, A. J. (12.122.060); Lesh, J. R. and Aizenman, M. L. (9.122.003); Levato, O. H. (8.116.011); Levato, H. (13.118.001); Levato, H. and Malaroda, S. (12.153.006); Levato, H. and Malaroda, S. (13.153.010); Lindoff, U. (9.153.005); Malaroda, S. (9.114.175); Mayer, P. and Macák, P. (9.114.026); MoCuskey, S. W., Pesch, P., and Snyder, G. A. (11.155.045); Moffat, A. F. J. (11.153.021); *Molnar, M. R. (8.114.009); *Morgan, W. W. and Abt, H. A. (9.114.144); *Morgan, W. W. and Keenan, P.C. (10.114.044); *Morgan, W. W., White, R. A., and Tapscott, J. W. (9.114.128); Naftilan, S. A. (13.121.063); *Newell, E. B. (10.115.030); *Oke, J. B. and Searle, L. (12.125.023); Pesch, P. (8.114.098); Pesch, P. and McCuskey, S. W. (11.155.021); *Preston, G. W. (12.065.061); Racine, R. (12.153.014); Richer, H. B., Sharpless, S. and Campbell, B. (8.152.009); *Rosendhal, J. D. (11.114.012); Schild, R. E. (9.114.050); *Schild, R. (9.114.019); Schultz, G.V. and Wiemer, W. (8.114.024); Sharpless, S. (12.152.008); Slettebak, A. (13.114.024); *Slettebak, A., Collins, G. W., Boyce, P. B., White, N. M., and Parkinson, T. D. (13.116.012); Smith, M. A. (8.152.002); Stephenson, C. B. (10.114.184); Stephenson, C. B. (12.114.033); Strom, S. E., Strom, K. M., and Carrasco, L. (12.153.033); Sturch, C. R. and Sharpless, S. (8.114.081); Swings, J. P. (10.133.069); Thackeray, A. D. and Andrews, P. J. (12.041.003); Thackeray, A. D., Tritton, S. B., and Walker, E. N. (10.112.003); Vreux, J. M. and Andrillat, Y. (12.114.031); *Walborn, N. R. (10.155.069); Walborn, N. R. (9.152.001); Walborn, N. R. (9.114.041); *Walborn, N. R. (11.114.079); Walker, M. F. (7.114.122); Webster, B. L. (12.113.027); *Webster, B. L. and Glass, I. S. (11.114.017); Wegner, G. (10.126.006); Wegner, G. (10.126.015); Wegner, G. (13.126.014); *Wolff, S. C. and Wolff, R. J. (12.114.107); Wyckoff, S. and Wehinger, P. (8.122.014); Wyckoff, S. and Wehinger, P. (10.114.182); Wyckoff, S. and Wehinger,P. (12.141.615); *Yamashita, Y. (8.114.130); Yamashita, Y. (13.114.072); *Yamashita, Y. (13.114.078); Young, A. and Martin, A.E. (9.153.030); Zinn, R. (9.114.148); Zinn, R. (9.114.139).

Work in Progress (extremely incomplete):

Bidelman and Lee (Warner and Swasey) are classifying some of the previously unstudied high-velocity stars of the Lowell Observatory northern hemisphere proper-motion survey. Bidelman also hopes to prepare for publication the many yet-unpublished spectral types of high-proper-motion stars determined by the late G. P. Kuiper many years ago. 
A. Cowley (U. of Michigan) has in press spectral classifications for some 575 bright F-type stars.

Garrison (David Dunlap) has completed observations of the 1300 brightest (to 4.75 mag.) stars in both hemispheres at 120,67 , and $12 \AA \mathrm{mm}^{-1}$ for the purpose of providing homogeneous classifications for these objects. He has also completed the classification of all southern OB stars brighter than 10th mag. (1200 stars). In addition the OB survey has been extended beyond 10 th mag. in some selected regions and observations are approximately $1 / 3$ complete for the classification of the integrated spectra of globular clusters.

Humphreys (Univ. of Minnesota) has in press a study of five of the bright variables in M 31 and M 33; all stars have emission-line spectra and that of one, AE And, bears a strong resemblance to that of $\eta$ Carinae.

Walborn (Cerro Tololo) is currently surveying the yellow-red region in OB spectra and is also engaged in classifying the $\mathrm{O}$ and $\mathrm{BO}$ supergiants of the Magellanic Clouds.

P. Dubois, M. and C. Jaschek (Strasbourg) are classifying the B-F supergiants of the Small Magellanic Cloud. Ch. Fehrenbach (Haute Provence) and the Jascheks are classifying the A-type supergiants in the Large Magellanic Clouds.

\section{OBJECTIVE PRISM SPECTRA AND AUTOMATED SPECTRAL CLASSIFICATION (E. K. Kharadze)}

Objective prism surveys in regions centred on the North and the South Galactic Poles have been undertaken at Abastumani (U.S.S.R.) and Perkins Observatory (U.S.A.).

Two-dimensional MK-classification of 1141 stars, down to the 11 th mag. (dispersions $166 \AA \mathrm{mm}^{-1}$ at $\mathrm{H \gamma}$ ), in 49 high galactic latitude regions of the MacCormick Proper Motion Catalogue has been carried out. The data are ready for publication (R. A. Bartaya, E. K. Kharadze, Abastumani Observatory).

An objective prism survey $\left(580 \AA \mathrm{mm}^{-1}\right)$ of G5-M stars in a 200 square degree region centered on the South Galactic Pole is being made (R. McNeil, Ohio State). The purpose of both works is the determination of the space density of stars as a function of distance from the galactic plane.

A system of three-dimensional spectrophotometric classification of objective-prism spectra $\left(250 \AA \mathrm{mm}^{-1}\right)$ has been established by A. Strobel (N. Copernicus University, Torun, Poland). The criteria for spectral class, luminosity class and $\mathrm{Fe} / \mathrm{H}$ content are based on measured ratios of depths of lines suitably chosen (Acta Astronomica, in press).

A method of numerical registration of spectra with computer-made transformation of the density scale into intensity on magnitude scale has been developed ( $Z$. Turlo, Bull. Astron. Torun, No. 53, 1975).

Rudolph, Schmidt-Kaler and Unger (Ruhr-University, Bochum) have continued their work on automatic two-dimensional spectral classification of low dispersion objective prism spectra (ca. 80-100 $\AA \mathrm{mm}^{-1}$ ). Early type stars (O-F5) can be classified automatically from low dispersion $\left(600 \AA \mathrm{mm}^{-1}\right)$ plates, using the Balmer jump and several line intensities, with an accuracy of about \pm 2 spectral subclasses and \pm 1 luminosity class.

West (III European Meeting, Tbilissi 1975) reports encouraging progress for automated multidimensional spectral classification, from plates having a dispersion of $166 \AA \mathrm{mm}^{-1}$, at the facilities of ESO in Geneva.

According to information from the Kitt Peak National Observatory (Hoag), low dispersion slitless spectra produced by transmission grating in the converging beam near the focal surface have proved to be useful for rough classification and searches for special objects to faint limits. Faint O-B stars in the Milky Way fields have been identified (Graham and Miller, 12.034.072; McCarthy and Miller, 09.113.017; Miller, 10.113.043). Be stars have been identified in a globular cluster (Bond, 10.114.049). Detection of faint $M$-stars in the galactic center and beyond (Blanco and Hoag), a search for faint emission-line objects and radio source identifications in the Hercules cluster of galaxies (Hoag) and a search for CH stars in globular clusters (Bond) are in progress. 


\section{CLASSIFICATION EMPLOYING MULTI-COLOUR WORK} (B. Hauck)

The multiplicity of photometric systems is a source of dispersion and loss of information, and books giving a summary of the properties, calibrations and applications of the various systems are very useful. The period covered has seen the publication of two such books, one by Golay (Introduction to Astronomical Photometry, D. Reidel Publ. 1974) and the other by Philip and Hayes ('Multi-Color Photometry and the Theoretical HR Diagram', Dudley Observatory Report No. 9, 1975, hereafter called the Albany Colloquium). A review paper was given by Bell at the Morgan Symposium (Lausanne, July 1975) on the derivation of abundances through photometric and spectroscopic methods.

The relations between indices of some systems were studied by different authors. Straizys (10.113.048) gives relations concerning the Vilnius system on the one hand and the $U B V$ and $u v b y$ systems on the other hand. Relations between the $U B V$ and $u v b y$ systems are studied in 10.133.088, while the comparison of the Geneva and uvby systems is given in 9.113.55. At the Albany Colloquium a study of the relations between $B 2-V 1$ and some temperature parameters of other photometric systems was presented, while the effect of the chemical composition on these relations was given at the Morgan Symposium. Also at the Albany Colloquium a comparison of $U B V R I$, DDO and $g n k f m n$ systems was given.

Theoretical colours were calculated for some systems and special kinds of stars. The $U B V$ colours for Population II giants (12.065.109), the $U B V$ and $u v b y$ colours for the $F$ and $G$ supergiants $(12.114 .058)$ and the Geneva colours for $5000 \leqslant T_{\text {eff }} \leqslant 8500 \mathrm{~K}, 2 \leqslant \log g \leqslant 4.5$ and $\chi=0,0.1$, and 1 (12.064.006 and Astron. Astrophys. 38, 417, 1975).

Steinlin and Buser (see Albany Colloquium) investigate the following questions:

Which problems concerning the determination of physical properties of stars can be solved by multi-colour photometry?

How have multi-colour systems to be designed to fulfil their purpose in an optimal way?

Evidence for a rotational reddening in early B-type stars is studied by Maeder (Astron. Astrophys., in press). Rapid rotation produces an intrinsic reddening of about 0.04-0.06 mag.

\section{A. Wide-Band Systems}

The UBVRI system has been used on many occasions. Feinstein (12.113.038) gives the data for 103 Am stars and studies the blanketing effect at different wavelengths, finding it important on $U$ and smaller and nearly the same in $B, V, R$ and $I$. Glagolevskij (10.114.050) shows that the relation between $T_{\text {eff }}$, determined from the energy distribution and the colour indices in the $U B V$ system proved to be the same for the Am and normal stars.

$U B V$ data and objective-prism spectral types have been determined for 62 stars in two regions at high galactic latitude $(09.113 .018)$ while a comparison is made of the $U B V$ photometry with spectral types for $145 \mathrm{G}$ and $\mathrm{K}$ giants near the NGP (09.114.050).

The difference of $U B V R I$ colours of strong-line $G$ dwarfs, subgiants and normal stars is studied in (09.113.032). In an $M_{v}$ vs $R-I$ diagram (10.115.005) strong-line $\mathrm{G}$ and $\mathrm{K}$ dwarfs show an unexpectedly large scatter.

Using the $V$ vs $R-I$ diagram for 56 stars located in the central region of the Hyades cluster, Upgren (12.153.025) derives for this cluster a distance modulus of $3.22 \pm 0.04$ mag. The gap present in the $U-B$ vs $B-V$ diagram is observed for field stars and for many clusters and must be related with the rotational velocities of the stars with $0.21<B-V<0.28$, the large rotational velocities shifting the onset of convection to lower $T_{\text {eff }}(12.153 .037)$.

The luminosity and velocity distribution of high-luminosity red stars was discussed by Eggen in a set of papers $(09.155 .009,10.113 .027,10.115 .037,11.115 .006$ and 12.115.018) while the luminosities and motions of the peculiar B-type stars are studied in (11.115.016). A study of the $M_{\text {bol }}$ vs $R-I$ plane for the subdwarfs $(9.126 .030)$ supports the conclusion that there is no continuity of main sequences between the Hyades stars and the subdwarfs.

Chaturvedi has used $X=\left[(B-V)^{2}+(V-R)^{2}\right]^{1 / 2}$ as temperature parameter for stars later 
than G0 (07.115.004) and $Z=(U+B)-(R+I)$ as luminosity criterion (11.115.003). On the basis of photographic data concerning four globular clusters, Rusev (11.113.007) demonstrated that the $I$ magnitudes and $B-I$ index are respectively luminosity and effective temperature criteria for red giants with $B-V \geqslant 1.5$ mag. $H, K$ and $L(1.65,2.2$ and $3.5 \mu)$ are given in (12.115.007) for $M$ dwarfs. This study suggests that the same blanketing function applies to all M dwarfs. By means of $(\mathbf{H}-\mathrm{K})$, WC stars were found systematically redder than WN stars (09.113.001).

A photometry at $20 \mu$ was developed (10.113.092) and data for 76 stars are given. A comparison of A General Catalogue of Cool Carbon Stars and The Two-Micron Sky Survey permitted the identification of seven sources of the latter (12.114.114).

The intrinsic ultraviolet colours $\left(U_{i}-V\right)_{0}$ are given for stars between B0 and $\mathrm{G} 3$ by Haramundanis and Payne-Gaposchkin (09.113.047) while extensive work on classification of various kinds of stars was made by Peytremann and Davis (12.113.031). Calibrations of $\left(U_{\mathrm{i}}-V\right)_{0}$ are also given. A discussion of the UV and UBV colours and MK spectral types for Ap stars was made by Lekrone (10.113.49) which showed a good agreement between UV and MK classes, but a disagreement with $U B V$ colours, the Ap being too blue.

Two UV multiband photometric systems have been carried out by Golay and his associates in view of extending, in the range 2000-3500 $\AA$, the Geneva system. Accurate photometric data have been obtained with a photoelectric photometer carried up to an elevation of $40 \mathrm{~km}$ by a stabilized balloon-borne telescope. The first is a 6 -intermediate band photometric system; the half-width is $150 \AA$ and the mean wavelengths are $2130 \AA, 2280 \AA, 2620 \AA, 2860 \AA$, and $3040 \AA$ The second is an eight-colour photometric system. Two colours are $B_{1}$ and $V_{1}$ from the seven-colour system $U B V B_{1} B_{2} V_{1} G$ (Geneva). The six others are obtained with narrow bands interference filters, $50-\AA$ bandwidth, and mean wavelengths, $2045 \AA, 2160 \AA, 2727 \AA$, $2955 \AA, 3067 \AA$, and $3355 \AA$. Normal stars, peculiar stars, stars with high $v \sin i$, and member stars of the Pleiades have been observed.

The Geneva system was employed in different ways. Maeder and Cramer have studied a luminosity calibration for B-type stars (Albany Colloquium) while work by Goy on O stars is in progress. The properties of the Ap stars and the possibility to detect them were investigated by Hauck (11.114.006, 11.113.031 and Colloquium No. 35 on Ap stars). $\Delta(V 1-G)$ is the parameter used to segregate the Ap stars from the normal and Am stars. The possibility to detect the $A-F$ supergiants was reported at the Albany Colloquium.

The calibration of the Geneva system for the late-type stars has been improved by Grenon, and over-all metal abundances, absolute magnitudes, $T_{\text {eff }}$, and photometric spectral types are now available for about $1200 \mathrm{G} 5$ to $\mathrm{K5}$ stars brighter than the 13 th mag. A special effort has been performed for the measurement of nearby stars. Moreover, a bi-dimensional classification is obtained for the $M$ stars, and peculiarities can be detected for the dwarf ones.

The $U B V B_{1} B_{2} V_{1} G$ catalogue of the intermediate bands photometric system contains (1974) 4671 very well measured stars (standard deviation: less than 0.01 mag.). In two previous papers, Golay has assumed that two stars having their seven colours identical within 0.01 mag. could be considered as being identical in effective temperature, chemical composition, absolute magnitude and interstellar reddening.

All stars having the seven colours identical within 0.01 mag. with a given star are members of a box. Inside a box, the spread of the spectral class reaches \pm 2 subclasses and \pm 1 luminosity class. The boxes containing Am stars are very homogeneous and almost all stars included in a box are Am stars. Peculiar stars and normal stars can be in a same box. Nevertheless, when we obtained - from spectroscopists - a re-examination of the spectrum of a normal star member of a box, which also contains an Ap star, some peculiarities were also discovered in the normal star. Among the 4671 stars of the last catalogue to be published, there are 1303 stars, members of at least one box. The spectroscopic observation, with several dispersions, has been undertaken in Haute-Provence by Ch. Fehrenbach, Y. Andrillat, and M. and C. Jaschek.

Fracassini et al. (09.115.025) have used the Geneva catalogue to obtain for $172 \mathrm{~B} \overline{5} \mathrm{~V}-\mathrm{A} 5 \mathrm{~V}$ stars their diameter. More recent work concerns 116 A5V-F5V stars (Fracassini, Pasinetti, and Pellagatti, Astrophys. Space Sci. 35, 313, 1975). 


\section{B. Medium-Band Systems}

\section{The Vilnius photometric system (report by V. Straizys)}

The new method for classification of stars in temperatures and gravities was proposed by V. Straizys (12.113.040) using the reddening-free energy distribution curves obtained either by photoelectric scanning or by multi-colour observations. The method is suitable also to identify the stars of different peculiarities (subdwarfs, metal-deficient giants, white dwarfs, metallic-line stars, carbon, barium and zirconium stars, multiple stars etc.) by seven colour photometry alone, without spectral observations and despite the presence of interstellar reddening.

The method is calibrated in temperatures and $\log g$ for all spectral types from $O$ to $M$ using model stellar atmospheres.

The further catalogue of photoelectric photometry of 175 stars measured in the Vilnius photometric system and classified in two dimensions was published in (11.113.003). The measurement of the system standards in SA at $+30^{\circ}$ and $0^{\circ}$ is in progress.

72 metal-deficient stars were observed with the aim to investigate the possibility to separate the effects of luminosity and chemical composition (11.113.005 and 12.113.056). Twodimensional classification was made for 26 stars around NML Cyg up to $V=15$ (12.113.017). Bogdanoviz and Alksnis have used the Vilnius system for photographic two-dimensional classification of 369 stars in the region of open cluster NGC 6871-12.113.015.

Osborn (09.113.009) has used the DDO system to derive some information about five extremely metal-poor Population II giants. Extensive work on the photometry of moving-group stars has been carried out by Boyle and McClure (Publ. Astron. Soc. Pacific 87, 17, 1975), who use the cyanogen strength and absolute magnitude calibrations of the DDO system to discuss the assignment of the observed stars to moving groups.

Many works on calibration of the $u v b y \beta$ system have been published, some based on the use of model atmospheres (Olson, 11.113.017; Breger, 12.113.019; Osmer and Peterson 11.064.002; Philip, Albany Colloquium), others based on an empirical way by Crawford (Albany Colloquium and paper in press for the calibration of the F stars).

Philip continued four-colour observations of early-type stars. Photometric classification of blue horizontal-branch stars $(10.113 .071)$ and the determination of interstellar reddening (10.113.083) have been published. Secondary standards $(09.113 .011)$ and a series of papers on observations of open clusters (09.153.013), globular clusters $(09.154 .014,09.113 .025)$, and certain areas in our Galaxy (10.113.107) have been published. Possible stellar members of the Magellanic Stream (11.159.004) have been detected (10.152.013). A programme of deep spectroscopy and $u v b y \beta$ photometry is underway. Photometry of metal-deficient A-type stars was made $(09.113 .056)$.

Over 100 field horizontal-branch stars have been measured in the $u v b y \beta$ systems by Philip and his associates. $\log g, \theta_{e}$ diagrams have been constructed for these stars and blue horizontal-branch stars in globular clusters for comparison with the predictions of evolutionary theories.

Many Ap and Am stars were measured by Warren (09.113.023). Drilling and Pesch (09.113.019) have obtained data for early-type stars near $l=179^{\circ}$ and $b=-47^{\circ}$ and some Am, Ap, horizontal branch and $\lambda$-Boo type stars were found.

Olson (12.113.050) used this system to study the late-type stars, obtaining a segregation in luminosity in the $(u-v)$ vs $(b-y)$ plane.

Strömgren (10.114.140) has reviewed the problem and state of quantitative spectral calibration, and in collaboration with Grønbech and E. H. Olsen has used a four-colour simultaneous photometer and (in a large programme) has obtained an exceptional low mean error for (southern) standard stars of $00^{\mathrm{m}} 0063$ in $y, 0^{m} .0035$ in $b-y, 0.0043$ in $c_{1}$ and 0 m 0044 in $m_{1}$. Olsen has made $u v b y \beta$ photometry of about 120 visual double stars with the purpose of determining absolute visual magnitudes for $\mathrm{G}$ and $\mathrm{K}$ stars. Kjaergaard, Olsen and Wilson (Hale observatories) are studying the abundance dependence of $M_{v}(K)(07.117 .040)$. Hansen has investigated the calibration of absolute visual magnitudes in terms of the Copenhagen $n, k, m, f$ indices on the basis of trigonometric parallaxes (10.115.007). Hansen is 
studying the optimum narrow- or intermediate-band classification system for $\mathrm{G}$ and $\mathrm{K}$ stars by means of photoelectric scanning. Clausen is preparing an investigation of the determinations of the abundance parameter for $F$ stars by means of quantitative classification measurements of Schmidt objective prism spectra $\left(102 \AA^{-1} \mathrm{~mm}^{-1}\right.$ at $\mathrm{H} \gamma$ ) (in continuation of 10.114.139).

\section{Narrow-Band Systems and Scans}

Further observations have been made on Wing's eight-color narrow-band photometric system, which measures near-infrared continuum colors and the strengths of $\mathrm{CN}$, TiO, and VO in late-type stars. The filter transmission functions and standard-star fluxes defining the system have been prepared for publication by Wing and Baumert (to be submitted to A stron. J.). Over 7000 sets of eight-color photometry of approximately 2000 different stars are now available (July 1975). These include nearly all stars of the following description: (1) red dwarfs within $10 \mathrm{pc}$ of the sun; (2) carbon stars with published proper motions; (3) M supergiants with published MK classifications; and (4) the 500 brightest $K$ and $M$ stars, in both hemispheres.

The extremely cool Mira variable IK Tauri has been studied by narrow-band photometry by Wing and Lockwood (10.122.043). Its spectral type is never earlier than $M 8$ and reaches $M 10$ at minimum more consistently than that of any other star known. Wing and Lockwood discuss the definitions of very late spectral types and recommend using $\mathrm{V} \mathrm{O}$ bands as the primary criterion after $\mathrm{M} 8$.

Wing $(10.122 .066)$ has obtained photometric classifications for red variables in the globular clusters $\omega$ Cen and 47 Tuc: Both clusters contain small-range variables as late as M 4 or M 5 , and the Mira variables in 47 Tuc reach at least type $M 7.5$ at minimum. The carbon stars belonging to $\omega$ Cen have been studied by Wing and Stock (10.114.248).

Two M supergiants belonging to the Large Magellanic Cloud have been classified photometrically by Wing (10.114.103). Their $\mathrm{CN}$ strengths confirm that they are supergiants but are somewhat weaker than those of stars of similar absolute magnitude in the Galaxy.

Color temperatures and photometric classifications have been obtained for approximately $150 \mathrm{M}$ dwarfs by Wing and Dean (in preparation). Kapteyn's star, a high-velocity M 0 subdwarf, is found to fall 1.4 bolometric magnitudes below the mean main sequence for stars within $10 \mathrm{pc}$ of the Sun (Wing, Dean, and MacCornell, submitted to Astrophys. J.).

Wing has established a six-color photometric system capable of yielding carbon isotope ratios for $\mathrm{G}$ and $\mathrm{K}$ stars as well as carbon stars (Highlights of Astronomy $3,285,1974$ ). It employs a spectral scanner $(20 \AA$ band pass) and measures the shape of the $\Delta v=+2$ sequence of the red $\mathrm{CN}$ system. More than 100 stars, mostly of high luminosity, have been observed on this system by Wing, Little, and Little-Marenin (in preparation).

The use of data concerning the $\mathrm{O}, \mathrm{B}, \mathrm{A}$ and $\mathrm{F}$ stars measured in the Barbier-Morguleff system has permitted the study of the Balmer discontinuity for the Ap stars (11.114.006). These data are now published (Astron. Astrophys. 19, 189, 1975). The analysis of the radiation between $3575 \AA$ and $3780 \AA$ has put in evidence the importance of the absorption by the metals in the Am stars. $35 \mathrm{Am}$ stars have been studied by Gerbaldi, which shows the important blocking effect in this spectral interval.

Chalonge and Divan (09.115.003) have given new calibrations of $M_{v}$ from the BCD system.

Faber (09.113.046) has developed a ten-colour system to study old stellar populations in globular clusters and galaxies. The system combines in fact five filters from the DDO system and six from Wood's 12-colour system.

Williams (Monthly Notices Roy. Astron. Soc. 170, 343, 1975) has obtained from his narrow-band photometry barium abundances for $200 \mathrm{~K}$ giants. Nissen (12.114.103) made photoelectric observations of a narrow-band index of the He I $\lambda 4026$ absorption lines for 148 main-sequence $B$ stars. He found that the absolute mean helium-to-hydrogen ratio for field stars is 0.08. Kjaergaard (12.114.011) together with Andersen and Gustafsson, has determined microturbulence and metal content in G8-K3 giants by means of photometry in very narrow bands and found that the microturbulence is remarkably constant for these stars. Similar work is in progress for $\mathrm{G}$ dwarfs by Kjaergaard and Melson.

Maitzen has defined a degree of peculiarity for Ap stars determined from photoelectric 
narrow-band photometry (2nd European Regional Meeting in Astronomy, in press, and IAU Coll. 35, in press).

Narrow-band photometry of individual hydrogen lines has been carried out. $\mathrm{H} \beta$ is frequently measured together with the $u v b y$ system and the parameter $\beta$ is described by Crawford (Albany Coll.) as temperature parameter for the $A$ and $F$ stars and as luminosity parameter for the $B$ stars. Feinstein (12.114.059) has established a system for $\mathrm{H} \alpha, \mathrm{H} \beta$ and $\mathrm{H} \gamma$ for 300 stars, especially designed to study the Balmer emissions in early type stars. He found that for stars of the same spectral type there exist differences in the behaviour of the normal and the emission line objects which can be attributed to large stellar rotation (IAU Symp. 70). Crawford et al. (Publ. Astron. Soc. Pacific 87, 115, 1975) have compiled $\mathrm{H} \alpha, \mathrm{H} \beta, \mathrm{H} \gamma$ and four-colour data for 780 B-type stars and analysed the relation between indices with respect to possible effects due to emission in the hydrogen lines. They found the possibility of separating supergiants from Be-type stars by means of an $H \alpha$ vs $H \beta$ diagram.

A system of standard stars for photoelectric $\mathrm{H} \alpha$ line photometry with narrow-band interference filters has been established by J. Dachs and Th. Schmidt-Kaler (Astron. Astrophys. Suppl. 21, 81, 1975). Two-dimensional calibration of Crawford's $\beta$ index of $H \beta$ line photometry in terms of both spectral type and luminosity class or $M_{v},(B-V)_{0}$ respectively, has been performed by Schmidt-Kaler and Klinkmann (1975, in preparation).

Sorvari (12.115.017) has defined a photometric parameter $r(\mathrm{O} I)$, based on the strength of the $O$ I feature at 7771,7774 and $7775 \AA$, to study A- and F-type stars. He found that $r(\mathrm{O} I)$ is temperature-sensitive in A-type supergiants and luminosity-sensitive in F-type supergiants. Using stars in galactic clusters he gives an absolute magnitude calibration for the latter. Another study of this spectral feature is made by Baker (11.115.005) which gives also a calibration in absolute magnitude for the F-type supergiants.

Measurements of $\mathrm{H} \gamma$ can be found in (11.114.170, 11.153.012 and 12.114.001) while a calibration in absolute magnitude is made by Balona and Crampton (11.115.002).

Honeycutt et al. (11.114.098) have derived from spectral scans a red colour index $(0.57)-(0.68) \mu$ for 32 carbon stars. This index is well correlated with the Yamashita spectral classes, except those with a (3.5)-(11.0) $\mu$ index greater than 0.7 .

Chaturvedi and Sinvhal (12.114.006) have used spectral scans to derive a colour index $\mathrm{C}=m_{5000}-m_{7000}$ as luminosity indicator for $\mathrm{K} 0-\mathrm{K} 5$ stars.

A photoelectric rapid-scanning single-channel spectrophotometer has been put into operation at the $61-\mathrm{cm}$ reflector of the University of Bochum installed at the European Southern Observatory, La Silla, Chile. The maximum resolution obtained with the scanner is $1 \AA$ in the second order of the grating $(3000-5000 \AA)$ and $2 \AA$ in the first order $(5000-8000 \AA)$. The instrument will be described in a forthcoming paper by Desjardins, Haupt, Maitzen, Rudolph, Schlosser and Schmidt-Kaler (1975, in preparation).

\section{ATLASES AND CATALOGUES \\ (C. Jaschek)}

\section{A. Atlases}

Albers (11.114.103) provides illustrations of the spectra which can be classified in the infrared $(7000-9000 \AA)$ at dispersions of $700 \AA \mathrm{mm}^{-1}$. Although the late type stars are the most suitable candidates, a number of other objects may as well be recognized.

An illustration of spectra taken at $1250 \AA \mathrm{mm}^{-1}$ is given by Miller and Graham (12.034.072).

Houk, Irvine and Rosenbush (1974) have published an Atlas to accompany the "Michigan Catalogue of two-dimensional spectral types for the Henry Draper Stars' at $108 \AA \mathrm{mm}^{-1}$, from objective prism plate material. About 80 spectra are reproduced.

Slettebak et al. (Astrophys. J. Suppl. 29, 137, 1975) have published an Atlas of rotationally broadened spectra, taken at a dispersion of $9 \AA \mathrm{mm}^{-1}$. This Atlas fills a long-standing gap in visual spectroscopy.

Seitter (Veroeffentl. Bonn, 1975) in continuation of part I of her Atlas, which illustrated OP 
spectra at $240 \AA \mathrm{mm}^{-1}$, has published the second part of the Atlas which corresponds to dispersions of 645 and $1280 \AA \mathrm{mm}^{-1}$. This Atlas is the first systematic illustration of the variation of classification parameters over a wide range in dispersion.

Keenan reports that an Atlas of MK spectral types for stars of types later than G2 is in preparation. For these late-type stars it will constitute a revision and extension of the Yerkes Atlas of 1943. The approximately 30 sheets of enlargements are based on negatives made with grating spectrographs at about $80 \AA \mathrm{mm}^{-1}$. It is planned to include with the enlargements a new list of standard type-stars. One essential purpose of the Atlas is to extend the MK-system to include abundance indices for stars in which composition differences can be detected on spectrograms of moderate dispersion. The Atlas should be ready for distribution by autumn 1976. It should be added that the IAU has contributed with a grant to this publication.

Osawa, Yamashita, Nariai and Norimito are preparing for publication an Atlas of representative stellar spectra, taken with a dispersion of $73 \AA \mathrm{mm}^{-1}$ at $\mathrm{H} \gamma$. The Atlas will contain about 40 sheets of standard spectra and 25 sheets of peculiar spectra.

\section{B. Catalogues}

We will consider under this title two different kinds of catalogues, namely:

Type (a) - lists of objects whose observed parameters were drawn from different sources. (If the catalogue includes unpublished results, these do not constitute the main contribution.)

Type (b) - long lists of systematic observations.

In what follows we have given a list of the most important catalogues of both kinds published lately.

\section{Catalogues Type (a), Spectroscopic}

A new general $O$ type stars catalogue - G. Goy - 10.114.113.

See also: A supplementary list of Southern O stars - A. D. Thackeray and P. J. Andrews 12.041.103.

A catalogue of galactic $O$ stars and the ionization of the low density interstellar medium by runaway stars - C. Cruz-Gonsales, E. Recillas-Cruz, R. Costero, M. Peimbert, and S. TorresPeimbert - R. Mex. A.A. 1, 211, 1974.

The evolutionary status of the blue halo stars - E. B. Newell - 10.115.030.

New catalogue of A stars with peculiar spectra (Ap) and with metallic lines (Am) Ch. Bertaud and M. Floquet - 11.114.097.

Catalogue of Am stars with known spectral types - B. Hauck - 09.114.140.

A general catalogue of cool carbon stars - C. B. Stephenson - 11.114.086.

A catalogue of $[\mathrm{Fe} / \mathrm{H}]$ determinations - M. Morel, C. Bentolila, G. Cayrel, and B. Hauck (in press).

MK spectral types by P. Kennedy, third edition, updated to 1975. On magnetic tape, available at the Centre de Données Stellaires, Strasbourg.

Stephenson prepares also a general catalogue of S-type stars, similar in scope to the one of cool carbon stars. In connection with this undertaking, Stephenson is observing the Northern Milky Way zones of the Hamburg-Cleveland survey, but with a red-region OP. Together with the Southern Sanduleak-Stephenson survey this will provide a homogeneous sky survey down to $m_{v} \sim 10-11$.

\section{Catalogues Type (b), Spectroscopic}

The brighter stars of astrophysical interest in the southern sky-W.P. Bidelman, D. J. MacConnell - 10.114.112.

University of Michigan Catalogue of two-dimensional spectral types for the HD stars, Vol. I - N. Houk and A. P. Cowley (1974) Vol. II, $-53^{\circ}$ to $-30^{\circ}$, is in preparation.

Low dispersion spectra and galactic distribution of various interesting strong-emission-line objects in the Southern Milky Way - N. Sanduleak, C. B. Stephenson - 10.114.152. 
The nature of the faint blue stars in the halo. II - J. R. Greenstein and A. I. Sargent 12.114.061.

A photometric and spectroscopic survey of large proper-motion stars - A. W. Rodgers and O. J. Eggen - 12.113.045.

Rotational velocities and spectral types for a sample of binary systems $-\mathrm{H}$. Levato $-A$ stron. Astrophys. Suppl. 19, 91, 1975.

A system of standard stars for rotational velocity determinations - A. Slettebak et al. - Astrophys. Suppl. 29, 137, 1975.

MK classifications for F and G type stars III - E. A. Harlan - 11.114.152.

Study of the F-type stars I. MK spectral types - S. Malaroda - Astron. J. 80, 637, 1975.

MK spectral types for some bright F stars - A. Cowley and D. Fraquelli - 11.114.063.

Catalogue of southern stars embedded in nebulosity - S. van den Bergh and W. Herbst Astron. J. 80, 208, 1975.

R. Associations I. $U B V$ photometry and MK spectroscopy of stars in southern reflection nebulae - W. Herbst - Astron. J. 80, 212, 1975.

Spectral types for proper motion stars - W. P. Bidelman and S. G. Lee - Astron. J. 80, 239, 1975.

The luminosity law for late-type main-sequence stars in the solar neighborhood - D. J. Eggen

$-12.115 .015$.

The subgiant $\mathrm{CH}$ stars $-\mathrm{H}$. E. Bond - 12.114.110.

Revised Catalogue of spectra of Mira type variables of type Me and Se - P. C. Keenan, R. F. Garrison and A. J. Deutsch - 12.114.048.

Spectral types of M dwarf stars - A. H. Joy and H. A. Abt - 12.114.035.

Catalogue of stellar magnitudes, color indices, spectral classes and luminosities of 6593 stars near the galactic pole - D. G. Chipashvili - 10.113.021.

Catalogue of stellar magnitudes, color indices, spectral classes and luminosities of 4268 stars near the north galactic pole - E. I. Zajtseva - 10.113.022.

Catalogue of stellar magnitudes, color indices, spectral classes and luminosities of stars in eight Kapteyn Selected Areas - A. D. Chuadze - 10.113.023.

Catalogue of stellar magnitudes, color indices, spectral classes and luminosities in Orion M. S. Kazanasmas - 10.113.024.

Catalogue of stellar magnitudes, color indices, spectral classes and luminosities in Andromeda - N. A. Miskin - 10.113.025.

A spectral survey of the Southern Milky Way I: general description and catalogue I $\left(l=306^{\circ}-318^{\circ}\right)$ - A. Sundman, L. O. Loden and B. Nordström - 12.114.020.

Part II, by B. Nordström Astron. Astrophys. Suppl. 21, 193, 1975.

Part III is in press and parts IV and V are in preparation.

\section{Catalogues Type (a), Photometric}

Catalogue of measurements in the Cape $U B V$ photometry on magnetic tape - B. Nicolet Astron. Astrophys. Suppl. 1975.

uvby photoelectric photometric catalogue - E. Lindemann and B. Hauck - 10.113.003.

A catalogue of photometric sequences - N. Argue, B. J. Bok, and E. W. Miller - 10.113.113. See also: E. W. Miller and C. C. MacCarthy - 12.113.039.

A catalogue of $\mathrm{H} \gamma$ measures by R. M. Petrie, D. Crampton, A. Leir, and F. Younger 11.114.170.

Luminosities and motions of peculiar B-type stars. - O. J. Eggen - 11.115.016.

Multicolor photometry of metallic-line stars III. A photometric catalogue - A. Mendoza $-R$. Mex. A.A. 1, 175, 1974.

Luminosity and velocity distribution of high-luminosity red stars IV. The G-type giants O. J. Eggen - 11.115.006.

High luminosity red stars in or near galactic clusters. Paper I - O. J. Eggen - 12.115.018.

Luminosity and velocity distribution of high-luminosity stars near the sun II. The young disk giants - O. J. Eggen - 10.113.027. 
Luminosity and velocity distribution of high-luminosity stars near the sun III. Old diskpopulation giants - O. J. Eggen - 10.115.037.

On the relationship between the apparent magnitudes given in several catalogues and UBV system - F. Ochsenbein - 11,113.020.

Catalogue of UBV measurements and MK spectral types in open clusters - J. Cl. Mermilliod, Lausanne (in preparation).

New catalogue of stellar UBV photoelectric photometry - J. Cl. Mermilliod, Lausanne (in preparation).

Similar catalogues of the DDO system, the Vilnius system and Eggen's system are in preparation (Inf. Bull CDS No. 7, 40, 1974).

A catalogue on magnetic tape indicating the photometric system in which a star is recorded (at least 35000 stars in 30 systems) is prepared by P. Magnenat, Lausanne.

See also: Absolute spectral energy distributions of common stellar types $-\mathbf{R}$. W. O'Connell 10.114.241.

\section{Catalogues Type (b) Photometric}

Celescope catalogue of ultraviolet stellar observations. Magnetic tape version - R. J. Davis, W. A. Deutschman, K. L. Haramundanis - 10.113.112.

Photoelectric catalogue of $1030 \mathrm{BD}$ M-type stars located along the galactic equator H. Neckel - 12.113.023.

Catalogue of early-type stars measured in a narrow-band photometric system $-\mathrm{N}$. Morguleff and M. Gerbaldi - Astron. Astrophys. Suppl. 19, 189, 1975.

Photoelectric photometry of stars near the North Galactic Pole - L. Häggkvist, T. Oja 10.113.057.

Photoelectric photometry of stars in the system URXYZVS VII - Bartkevicius et al. -11.113 .004 .

Cyanogen strengths, luminosities and kinematics of $\mathrm{K}$ giant stars - K. A. Janes - Astrophys. Suppl. 29, 161, 1975.

Absolute spectral energy distributions for white dwarfs - J. B. Oke -11.126 .005 .

\section{REPORT OF THE GROUP FOR COLLECTION OF PHOTOMETRIC AND SPECTROSCOPIC DATA}

(B. Hauck)

At the administrative session of Commission 45 it was decided to give a new mandate to the members of the Group, with Dr K. Nandy becoming a new member. The composition of the Group is at present as follows:

M. Barbier, W. P. Bidelman, B. Hauck.(Chairman), C. Jaschek, K. Nandy, B. E. Westerlund.

During the period under consideration the publication of lists of recently published catalogues or those about to be published has been continued $(009.002 .007$ - $011.113 .041-$ No. 8, p. 17, 1975). Lists of catalogues on magnetic tape were in fact published by Hauck and Jung (12.041.002), by Jung and Guilbaut (11.041.074) and by Jaschek, et al. (Inf. Bull. CDS No. $8,22,1975$ ).

Another task of our. Group was the study of the possibility of publishing in an astrophysical journal an announcement of catalogues on magnetic tape. It is necessary that such catalogues be recognized as publications and the only way to obtain such recognition is to publish a short description in a journal. Consultation of members of the Group and Dr. Wilkins, Chairman of the Group on Numerical Data, and discussions with Drs Pottasch and Heidmann, Chief Editors of Astronomy and Astrophysics, have led to the publication of an editorial in the October 1974 issue of Astron. Astrophys. Supplement Series. Thus the possibility of such announcements now exists in accordance with certain well-defined rules.

With regard to the $I A U$ Symp. 35 (Compilation, Critical Evaluation and Distribution of 
Stellar Data, Strasbourg, August 1976), two members of our Group are also members of the Scientific Organizing Committee (Jaschek and Hauck). After consultation of the other members I have proposed that this colloquium should be concerned not only with stellar data but also with methodological problems related to all astronomical data.

\author{
C. JASCHEK \\ President of the Commission
}

